\title{
A Brief Review on Therapeutic Potential of Nanocarrier Systems Against Breast Cancer
}

\author{
Swarupananda $M^{*}$, Saumyakanti G, Subhasis M and Bijaya G \\ NSHM College of Pharmaceutical Technology, NSHM Knowledge Campus, Kolkata Group of Institutions, (60) 127 BL Saha Road, \\ Kolkata 700053, West Bengal, India
}

\begin{abstract}
Now a day's breast cancer is one of the major type of non-cutaneous cancer occurred in female bodies. There are many approaches to treat this type of cancer, but significant treatments for breast cancer are truly limited. Not only the existing treatments are very low in number, but they have number of toxicity issues also. That is why now nano carrier based approaches are under the spot light and researchers are trying to explore this novel area of treatment for breast cancer. The success of any anticancer chemotherapy truly depends on the ability of the drug to reach their targeted body site, where the abnormal cell growth has occurred. Conventional types of treatment has no such targeting benefits. The nanocarriers based drug delivery systems are having some great advantages. These carrier systems have great specific targeting ability; they can modify the bio-distributionand pharmacokinetic profiles of the therapeutic agents. That is why nanocarriers are having lesser cytotoxic side effects than the conventional type of treatments. And these benefits make these carrier systems unique. The popular nanocarries used for today are liposomes, carbon nanotubes, micelles, quantum dots, nanoparticles, gold nanoparticles, solid lipid nanoparticles etc. This review is a brief discussion about the nanocarriers used for breast cancer today and their advancements.
\end{abstract}

Keywords: Breast cancer; Nanocarriers; Liposomes; Nanosomes

\section{INTRODUCTION}

Breast cancer is one type of heterogeneous disease and it is having different type of subcategories. It is reported that approximately $7 \%$ of breast cancers occurred in women who are having age below than 40 years and less than $4 \%$ in women below the age of 35 years [1]. In young women breast cancer is not generally common [2]. Cancer is caused by damage or mutations. Generally surgery and radiotherapy are the primary treatments for local and nonmetastatic cancers while anti-cancer drugs (chemotherapy, hormone and biological therapies) are the choice of metastatic cancers. The basic principle of the chemotherapy is the inhibition of the division of rapidly growing cancer cells. But this therapy also affects normal cells those are having fast proliferation rates, such as the hair follicles, bone marrow and gastrointestinal tract cells, generating the characteristic side effects of chemotherapy. Nanocarriers are the small carrier molecules having size range between ten to hundred nanometers. These are mainly cluster of molecules, atoms and molecular fragments. The most popular nanocarries used for today are liposomes, carbon nanotubes, micelles, quantum dots, nanoparticles, gold nanoparticles, solid lipid nanoparticles etc.

\section{COMMON DRUG CARRIERS USED IN BREAST CANCER}

\section{Liposome}

Liposomes are one of the most common types of novel drug delivery system for breast cancer treatment (Figures 1 and 2). Liposomes can improve the pharmacological properties of the drugs by changing its distribution and pharmacokinetic profile in the body [3-5]. The liposomal-based chemotherapeutic agents have been passed the clinical trials successfully. And that is why these formulations are currently used for the treatment of the breast cancer. Doxil is one example of the approved liposomal preparations [6]. Liposomes loaded with chemotherapeutic drugs like doxorubicin (Doxil) and daunorubicin (DaunoXome) have been approved by FDA since the mid-1990s. Liposomes are also called as colloidal carriers and are made up of bio-compatible, bio-degradable, natural phosopholipids. We can encapsulate water-soluble, fat-soluble, amphiphilic, biphasic insoluble drugs and cytotoxic agents into their aqueous core or their unilamellar/ multilamellar membranes depending upon the solubility of the

Correspondence to: Swarupananda M, NSHM College of Pharmaceutical Technology, NSHM Knowledge Campus, Kolkata Group of Institutions, (60) 127 BL Saha Road, Kolkata 700053, West Bengal, India, E-mail: swarupananda.mukherjee@nshm.com

Received: February 01, 2019, Accepted: March 12, 2019, Published: March 20, 2019

Citation: Swarupananda M, Saumyakanti G, Subhasis M, Bijaya G (2019) A Brief Review on Therapeutic Potential of Nanocarrier Systems Against Breast Cancer. J Biomol Res Ther, 8: 174.

Copyright: $@ 2019$ Swarupananda M, et al. This is an open access article distributed under the term of the Creative Commons Attribution License, which permits unrestricted use, distribution, and reproduction in any medium, provided the original work is properly cited. 


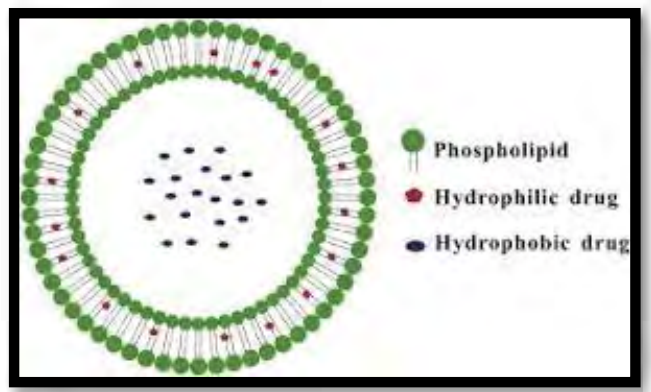

Figure 1: Principle construction of a liposome.

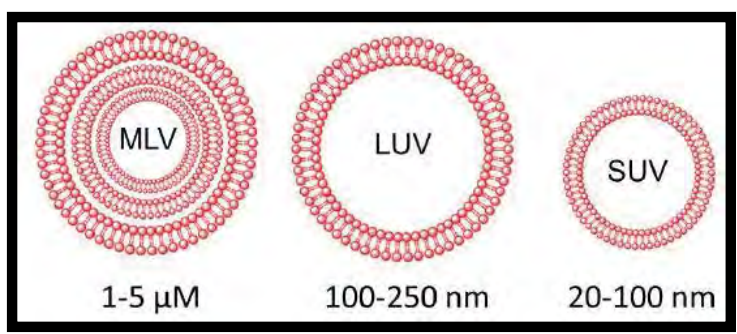

Figure 2: Different types of liposomes.

API. Liposomes have two parts, one is lipid bilayer membrane part and another is aqueous core part. Liposomes are generally classified into three types: Small Unilamellar Vesicles(SUV), Large Unilamellar Vesicles (LUV) and Multilamellar Vesicles (MLV) [7]. Liposomes are generally preferred for the following reasons. One is liposomes provide slow and sustained release, secondly they reduces cytotoxicity of the chemotherapeutic agents and thirdly they enhance the drug accumulation. New researches on liposomes are now based on modifying the drug release from liposomes. For example, drug release from liposomes can be triggered by various factors $[8,9]$. For example drug release can be triggered by ultrasound enzymes light, magnetism etc. [10-16]. Liposomes can also be attached with some ligands. These ligands basically acts for targeting purpose. Ligand mediated liposomes are used for the targeted delivery of nucleic acids [17-20]. Doxil isone of the classic example of nanocarriers used in breast cancer. It is constituted with cholesterol and Hydrogenated Soy Phosphatidylcholine (HSPC). HSPC is having high phase-transition temperature that leads to a stable drug delivery system with improved efficacy and reduced side effects [21]. Shahun had formulated one type of liposomes which can be loaded with Doxorubicin (DOX). This liposome can be targeted actively by using peptide ligands known as P18-4 [8]. Recently Dagar developed a vasoactive intestinal peptide receptors (VIP-R). This VIP-R is used in breast cancer for targeted imaging purpose. VIP-R is basically a mammalian neuropeptide which was attached to the surface of the Stericallystabilized Liposomes(SSL) covalently, which was further encapsulated to a radionuclide (Tc99. HMPAO). Further studies revealed that VIP-R is more expressive in case of human breast cancer by five times in comparison with other imaging probes [22].

\section{Micellar nanoparticles}

These are also a highly specific nanocarriers, consists of a core and a shell in structure (Figure 3). These are basically colloidal particles, which are having size between 5 to 100 nanometers. Micelles mainly consist of surfactants. Surfactants are amphiphilicin nature. They are having a polar head portion which is hydrophilic in nature. And another potion is no-polar, hydrophobic tail portion. These surfactants exist as monomers in low concentration. But when we increase the concentration of the surfactants gradually then above the critical micelle concentration (CMC) they form micelles. These micelles have a hydrophilic core, in which we can incorporate hydrophobic drugs. Micelles are very useful for targeting the in to the site of action. A classic example of micelles is Genexol-PM (approved by FDA in 2007) which is loaded with the anticancer drug paclitaxel [23-25]. It is composed of mPEG-PLA polymers. It showed not only greater targeting action but it showed to reduce the severe toxic effects such as hypersensitivity reactions, hyperlipidemia also.

\section{Dendrimer}

Origin of the word Dendrimeris from the Greek word "dendron", Dendron means 'tree-like structures'. Dendrimerswas synthesized by Buhleier et al. and Tomalia et al. from 1970-1990 [26,27]. There are different types of dendrimersbased on theirshape and size. They show higher biocompatibility. Chemical modification in the structure of dendrimerscould lead a better carrier. Recently, Wang had synthesized G4 Polyamidoaminedendrimer (G4 PAMAM-D). It is conjugated with Antisense Oligodeoxynucleotides (ASODN). This conjugated carrier system show greater stability in the body with less toxicity. It also showed increased bioavailability. Further in vivo studies revealed that this conjugated syatemhas more accumulating power to inhibit tumor than naked ASODN [28].

\section{Virus-based nanoparticles (VNPs)}

Virus-Like Particles (VLPs) are self-assembled robust protein cages having uniform nanostructures and well-defined geometry. Their size is approximately less than $100 \mathrm{~nm}$. Recently scientists are exploring VNPs widely. These VNPs are also used in various purposes excluding drug delivery. Viruses from different sources are used for VNPs. Recently it is found that plant viruses (Cowpea Mosaic Virus [CPMV], Red Clover Necrotic Mosaic Virus [RCNMV], Tobacco Mosaic Virus [TMV], Cowpea Chlorotic Mottle Virus [CCMV]), insect viruses (Flock House Virus), bacterial viruses or bacteriophages (MS2, M13, Q!) and animal viruses (adenovirus, polyomavirus) have the potential to act as a drug delivery carrier [29-31].

\section{Polymers}

Polymeric nanoparticles are also used for universal drug delivery carriers. These are formed by attaching a copolymer to a polymer matrix. Cellulose, chitosan, alginate, and gelatin are the most common polymers those are used for the preparation of polymeric nanoparticles. These polymers are having several advantages. The most important advantage is that they are biocompatible and biodegradable. They, also slow down the drug degradation rate, increase the drug stability. Chemotherapeutic drugs like paclitaxel,

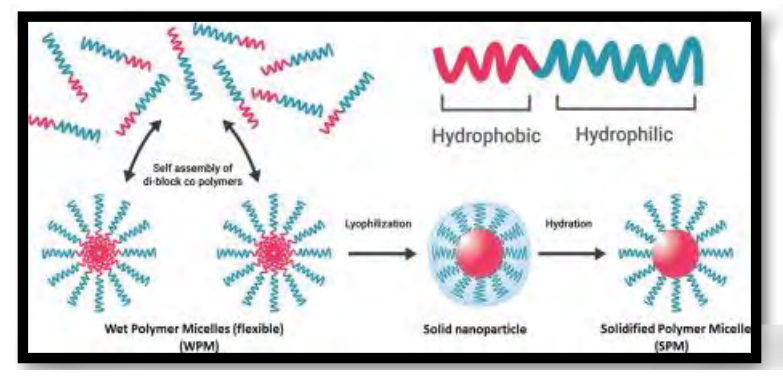

Figure 3: Different pattern of Micellar nanoparticles. 
doxorubicin, camptothecins, and platinates have been successfully clinically tested through these polymeric nanocarriers.

\section{Gold carriers}

Gold carriers have a core of gold atoms. These atoms could be functionalized by the attaching of unilayer of thiol (SH)-containing groups [32,33]. These carrier molecules are synthesized with the help of thiol-containing groups. These thiol containing groups actually forms a layer around the gold atoms. The first clinical trial with gold carriers done in 2009 [34].

\section{Magnetic carriers}

These are also a type of special carriers those are used in breast cancers (Figure 4). These are generally placed into the bloodstream of the patient and a magnetic field is focused over the target site [35]. The first clinical trial report came in 1996 [36].

\section{Albumin carriers}

Body protein albumin is also widely used as a drug carrier. It is having good solubility in both water and ethanol. Albumin is an integrated part of our own human body system that makes it nontoxic, well-tolerated and higher half-life in blood circulation [37]. The first anticancer drug containing albumin carrier is Abraxane, paclitaxel is attached to this carrier [38].

\section{Carbon carriers}

Carbon Nanotubes (CNTs) are the carriers those are the members of the fullerene family. Structurally CNTs are made up of benzene rings rolled-up into the tubular structure (Figure 5). CNTs are having two classes based on their structure. One is Single-Walled (SWNTs) CNTs, and another is Multi-Walled (MWNTs) CNTs. SWNTs are made up of one layer of cylinder graphene and MWNTs contains multiple concentric graphene layers. There are some extraordinary features of CNTs. They have well organized structure, very light weight, high mechanical strength, and high surface area [39]. These

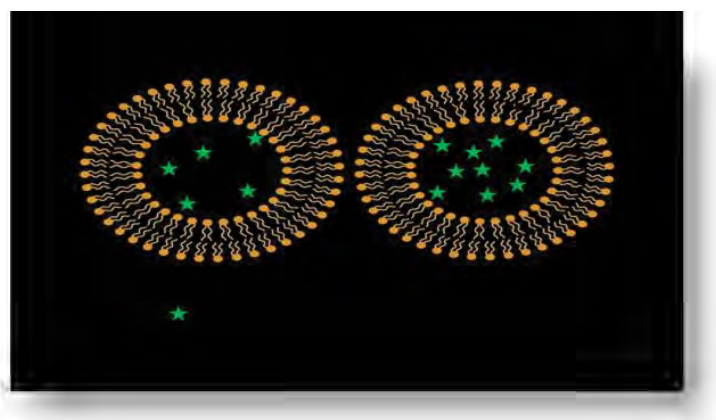

Figure 4: Magnetic nanoparticles.

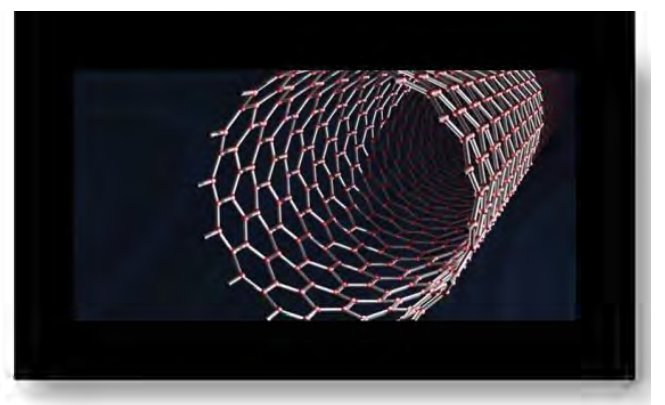

Figure 5: Carbon nanotube. properties make CNTs a good candidate for drug delivery in case of breast cancer [40].

\section{CONCLUSION}

Nanotechnology is relatively new in cancer treatment. It has a great potential in the treatment of different types of cancer including breast cancer and from the research it is very clear that nanocarriers are now one of the promising tools for cancer treatment and drug delivery. Still there are some limitations to use these nanocarriers. The targeting mechanisms of the nanocarriers and the development of multi-drug resistances should be investigated more carefully. In spite of these limitations there are numerous benefits of these nanocarriers and these advantages make them one of the most promising treatments for breast cancer.

\section{REFERENCES}

1. Brinton LA, Sherman ME, Carreon JD, Anderson WF. Recent trends in breast cancer among younger women in the United States. J Nat Cancer Inst. 2008;100:1643-1648.

2. https://seer.cancer.gov/archive/publications/aya/aya mono complete.pdf

3. Khan DR, Rezler EM, Lauer-Fields J, Fields GB. Effects of drug hydrophobicity on liposomal stability. Chem Biol \& Drug Des. 2008;7:3-7.

4. https://global.oup.com/academic/product/liposomes-a-practicalapproach -9780199636549 ? $\mathrm{cc}=\mathrm{in} \&$ lang $=\mathrm{en} \&$

5. Rezler EM, Khan DR, Lauer-Fields J, Cudic M, Baronas-Lowell D. Targeted drug delivery utilizing protein-like molecular architecture. J American Chem Soci. 2007;129:4961-4972.

6. Khan DR. The use of nanocarriers for drug delivery in cancer therapy J Cancer Sci \& Ther. 2010;2:58-62.

7. Hofheinz RD, Gnad-Vogt SU, Beyer U, Hochhaus A. Liposomal encapsulated anti-cancer drugs. Anti-Cancer Drugs. 2005;16:691-707.

8. Huang S-L, MacDonald RC. Acoustically active liposomes for drug encapsulation and ultrasound-triggered release. Biochim Biophys Acta. 2004;1665:134-141.

9. Ueno Y, Sonoda S, Suzuki R, Yokouchi M, Kawasoe Y. Combination of ultrasound and bubble liposome enhance the effect of doxorubicin and inhibit murine osteosarcoma growth. Cancer Biol Ther. 2011;12:270277.

10.Pak CC, Erukulla RK, Ahl PL, Janoff AS, Meers P. Elastase activated liposomal delivery to nucleated cells. Biochim Biophys Acta. 1999;1419:111-126.

11. Meers P. Enzyme-activated targeting of liposomes. Adv Drug Deliv Rev. 2001;53:265-272.

12. Gerasimov OV, Boomer JA, Qualls MM, Thompson DH. Cytosolic drug delivery using $\mathrm{pH}$ - and light-sensitive liposomes. Adv Drug Deliv Rev. 1999;38:317-338.

13. Bondurant B, Mueller A, O'Brien DF. Photoinitiated destabilization of sterically stabilized liposomes. Biochim Biophys Acta. 2011;1511:113 122.

14. Du B, Han S, Li H, Zhao F, Su X. Multi-functional liposomes showing radiofrequency-triggered release and magnetic resonance imaging for tumor multi-mechanism therapy. Nanoscale. 2006;7:5411-5426.

15.Arie AA, Lee JK. Effect of boron doped fullerene C 60 film coating on the electrochemical characteristics of silicon thin film anodes for lithium secondary batteries. Synth Met. 2011;161:158-165. 
16. Dao TT, Matsushima T, Murata H. Organic nonvolatile memory transistors based on fullerene and an electron-trapping polymer. Org Electron. 2012;13:2709-2715.

17. Landen CN Jr, Chavez-Reyes A, Bucana C, Schmandt R, Deavers MT. Therapeutic EphA2 gene targeting in vivo using neutral liposomal small interfering RNA delivery. Cancer Res. 2005;65:6910-6918.

18. Miller CR, Bondurant B, McLean SD, McGovern KA, O'Brien DF. Liposome-cell interactions in vitro: Effect of liposome surface charge on the binding and endocytosis of conventional and sterically stabilized liposomes. Biochemistry. 1998;37:12875-12883.

19. Wolfrum C, Shi S, Jayaprakash KN, Jayaraman M. Mechanisms and optimization of in vivo delivery of lipophilic siRNAs. Nat Biotechnol. 2007;25:1149-1157.

20.Wang Z, Yu Y, Dai W, Lu J, Cui J. The use of a tumor metastasis targeting peptide to deliver doxorubicin-containing liposomes to highly metastatic cancer. Biomaterials. 2012;33:8451-8460.

21. Elbayoumi TA, Torchilin VP. Tumor-specific antibody-mediated targeted delivery of Doxil reduces the manifestation of auricular erythema side effect in mice. Int J Pharmaceut. 2008;357:272-279.

22.Dagar S. VIP grafted sterically stabilized liposomes for targeted imaging of breast cancer: in vivo studies. J of Controlled Release. 2003;91:123133

23.Ahn HK, Jung M, Sym SJ, Shin DB, Kang SM. A phase II trial of Cremorphor EL-free paclitaxel (Genexol-PM) and gemcitabine in patients with advanced non-small cell lung cancer. Cancer Chemother Pharmacol. 74:277-282.

24.Park S, Healy KE. Nanoparticulate DNA packaging using terpolymers of poly (lysine-g-(lactide-b-ethylene glycol)). Bioconjug Chem. 2003;14:311319.

25.Xue Y, Tang X, Huang J, Zhang X. Anti-tumor efficacy of polymerplatinum(II) complex micelles fabricated from folate conjugated PEGgraft- $\alpha, \beta$-poly [(N-amino acidyl)-aspartamide] and cis-dichlorodiammine platinum(II) in tumor-bearing mice. Colloids \& Surfaces. 2011;85:280288.

26.Buhleier E, Wehner W, Vögtle F. Cascade'- and 'nonskid-chain-like' syntheses of molecular cavity topologies. Synthesis (Mass). 1978;6:155158.

27.27. Tomalia DA, Baker H, Dewald J, Hall M, Kallos G. A new class of polymers: Starburst-dendritic macromolecules. Polym J. 1985;17:117132.
28.Wang P, Zhao X-H, Wang Z-Y, Meng M, Li X. Generation 4 polyamidoamine dendrimers is a novel candidate of nano-carrier for gene delivery agents in breast cancer treatment. Cancer Letters. 2010;298:34-49.

29.29. Manchester M, Singh P. Virus-based nanoparticles (VNPs) platform technologies for diagnostic imaging. Adv Drug Deliv Rev 2006;58:1505-1522.

30.30. Singh P, Prasuhn D, Yeh RM, Destito G, Rae CS. Bio-distribution, toxicity and pathology of cowpea mosaic virus nanoparticles in vivo. J Control Release. 2007;120:41-50.

31. 31. Ma Y, Nolte RJ, Cornelissen JJ. Virus-based nanocarriers for drug delivery. Adv Drug Delivery Rev. 2012;64: 811-825.

32.32. Xu ZP, Zeng QH, Lu GQ, Yu A. Inorganic nanoparticles as carriers for efficient cellular delivery. Chem Engin Sci. 2006;61:1027-1040.

33.33. Connor EE, Mwamuka J, Gole A, Murphy CJ, Wyatt MD. Gold nanoparticles are taken up by human cells but do not cause acute cytotoxicity. Small. 2005;1:325-327.

34.34. Libutti SK, Paciotti GF, Myer L, Haynes R, Gannon W. Results of a completed phase I clinical trial of CYT-6091: A pegylated colloidal goldTNF nanomedicine. ASCO Ann Meet Proceed. 2009;4:3586.

35.35. Dobson J. Magnetic nanoparticles for gene and drug delivery. Int J Nanomed. 2008;3:169-180.

36.36. Lubbe AS, Bergemann C, Riess H, Schriever F, Reichardt P. Clinical experiences with magnetic drug targeting: a phase I study with 4'-epidoxorubicin in 14 patients with advanced solid tumors. Cancer Res. 1996;56:4686-4693.

37.37. Kratz F. Albumin as a drug carrier: design of prodrugs, drug conjugates and nanoparticles. J Control Release. 2008;132:171-183.

38.38. Hawkins MJ, Soon-Shiong P, Desai N. Protein nanoparticles as drug carriers in clinical medicine. Adv Drug Deliv Rev. 2008;60:876-885.

39.39. Lacerda L, Bianco A, Prato M, Kostarelos K. Carbon nanotubes as nanomedicines: From toxicology to pharmacology. Adv Drug Deliv Rev. 2006;58:1460-1470.

40.40. Jayakumar R, Prabaharan M, Nair SV, Tamura H. Novel chitin and chitosan nanofibers in biomedical applications. Biotechnol Adv. 2010;28:142-150. 